\title{
Measurements of D/H Ratio Using Compact Neutral Particle Analyzer in LHD Deuterium Experiments*)
}

\author{
Tetsuo OZAKI, Shuji KAMIO, Kenji SAITO and the LHD Group \\ National Institute for Fusion Science, 322-6 Oroshi, Toki 509-5292, Japan
}

(Received 12 January 2020 / Accepted 8 April 2020)

\begin{abstract}
The deuterium/hydrogen ratio in the plasma strongly affects against the plasma heating and other behaviors. The ratio in high energy region is measured by the charge exchange neutral particle analyzer instead of the spectroscopic technique. The ratio has obtained by reducing the electrostatic plate voltage in the compact neutral particle analyzer, which is tuned for hydrogen measurement. When the voltage is adjusted for deuterium, hydrogen simultaneously enters as contamination because the hydrogen orbit is closed to the mid-plane. Here, the mass rejection factor has been evaluated and the time variation of the ratio in the low and high energy regions during hydrogen and deuterium neutral beam injection is described. The behavior of the ratio in high and low energy regions can be explained by the classical slowing down process of the energetic particle.
\end{abstract}

(C) 2020 The Japan Society of Plasma Science and Nuclear Fusion Research

Keywords: CNPA, LHD, deuteron, hydrogen, $\mathrm{H}_{\alpha}, \mathrm{D}_{\alpha}$, spectroscopy, NBI, gas puff, wall, pellet

DOI: $10.1585 /$ pfr.15.2402034

\section{Introduction}

In a fusion reactor, the different hydrogen isotopes, deuterium and tritium, are used as fuel. Therefore, it is extremely important to understand the ratio of deuterium to tritium and their behavior in the future nuclear fusion reactor. In addition, in ion cyclotron resonance heating ( $\mathrm{ICH}$ ), the control of the particle species and the ratio are important because the energy distribution and spatial distribution of the ratio strongly depend on the location of the heating layer and the heating efficiency. In the Large Helical Device (LHD) [1], hydrogen, deuterium and helium are used to simulate those isotope effects.

The actual ratio of hydrogen to deuterium, $D /(D+H)$, is not a simple ratio of the input gas, but strongly depends on the location and energy. In particular, since hydrogen derived from water adsorbed on the wall of the vacuum vessel strongly contributes to the peripheral region of the plasma, even if deuterium is supplied by gas puff or fuel pellet, the hydrogen contribution becomes large in the peripheral region. This effect is remarkable in LHD because a helical device such as the LHD has a large surface area compared to the volume of plasma. When hydrogen or deuterium is supplied by the neutral beam injection (NBI), it becomes more complicated. Since the beam collides with gas/plasma, the ratio of hydrogen/deuterium changes with energy and time.

It takes a considerable time to obtain a complete deuterium plasma. Numerous deuterium glow discharges are required to obtain deuterium-dominated plasmas, since hydrogen is gradually replaced by deuterium gas puff, pellet

author'se-mail: ozaki@nifs.ac.jp

*) This article is based on the presentation at the 28th International Toki Conference on Plasma and Fusion Research (ITC28). injection and NBI.

Generally, the deuterium/hydrogen ratio is mainly monitored using a spectroscopic method [2]. However, since this method observes light emission $\left(\mathrm{H}_{\alpha}\right.$ or $\left.\mathrm{D}_{\alpha}\right)$ from excited neutral hydrogen or deuterium in a low temperature plasma, only information on extremely low energy and a peripheral plasma can be obtained. In LHD, the beam charge exchange spectroscopy using NBI is also utilized [3]. Although this method solves the problem of spatial distribution, the problem of the deuterium ratio in the high energy region still remains. A traditional method for measuring the deuterium/hydrogen ratio in the high energy region is to use a neutral particle analyzer. There is not only neutral hydrogen or deuterium supplied from gas puffs or NBI, but also neutral particles from the wall as charge exchange neutral source in plasma. Those neutrals are penetrated into the plasma center without the affect of the plasma confinement magnetic field by repeating the chain reaction of the charge exchange. As a result, the deuterium/hydrogen ratio in the relatively central region can be easily measured. Simple passive measurement does not provide spatial information because it is integrated in the line of sight. However, in the future it is possible to measure the spatial distribution of deuterium ratio in the high energy region by combining with a diagnostic beam or an impurity pellet [4-7].

In LHD, the deuterium/hydrogen ratio is varied by tangential and perpendicular direction because of the high energy particle sources such as NBI and ICH. Neutral particle analyzers [8] are also installed in the tangential direction, and it is interesting to compare the difference of the ratio between the tangential and perpendicular directions. Here, we describe the deuterium/hydrogen ratio measured 
by a compact neutral particle analyzer (CNPA) installed perpendicular to the plasma.

\section{Experimental Setup and Device}

LHD is a helical device with a major radius of $3.9 \mathrm{~m}$ and a small radius of $0.6 \mathrm{~m}$ with toroidal number $m=10$, poloidal number $l=2$. The main heating devices are an electron cyclotron heating, NBI and ICH. Three NBIs are installed in the tangential direction $(180 \mathrm{keV}$ for hydrogen and deuterium) and two in the vertical direction (40 $60 \mathrm{keV}$ for hydrogen and deuterium). Switching between hydrogen and deuterium in NBI particle species is performed weekly according to the experimental plan.

The tangential NBIs have a single energy of hydrogen or deuterium due to neutralization of the negative ion source. The perpendicular NBIs have hydrogen energies of $1 / 1,1 / 2$, and $1 / 3$ because the proton/deuteron beam is neutralized. Fuel is supplied by gas puff and ice pellets (hydrogen or deuterium). The ratio of deuterium/hydrogen is measured by photomultipliers using band-path filters of $\mathrm{H}_{\alpha}$ and $\mathrm{D}_{\alpha}$ and determined from those intensity ratios.

CNPA combined with the impurity pellet measurement is one of the few instruments that can directly measure the radial high energy particle distribution. CNPA is installed almost perpendicular to the equatorial plane of LHD, thus neutral particles from the perpendicular NBI and $\mathrm{ICH}$ can be mainly observed. Neutral particles originating from the tangential NBI are also observed in a considerable amount due to multiple pitch angle scattering. In the deuterium experiment, CNPA is shielded with $25 \mathrm{~cm}$ thick boron doped polyethylene from strong neutron flux. Remaining neutron noise is eliminated as follows. The correlation between neutron noise and the neutron yield measured by a scintillator photomultiplier [9] and ${ }^{235} \mathrm{U}$ fission chamber [10] etc., can be evaluated in advance when the gate valve is closed [11]. The correlation between the neutron noise and the neutron yield is in very good proportion over three orders of magnitude. Based on the correction, the amount of incident charge exchange neutral particles can be calculated.

Neutral particles are ionized by a thin carbon film, and those energies are separated by a permanent magnet in CNPA. The energy separated ions are deflected by an electric field. CNPA is designed only for measuring hydrogen. Therefore, it is necessary to change the deflection plate voltage to measure deuterium. The orbit of the proton with energy $E$ is same as the orbit of the deuteron with $E / 2$ in the equatorial plane in the same magnetic field.

The ions coming out of the magnet are discriminated by the deflection plate. Since the deflection is determined by the charge of the particle, it is the same in the proton and the deuteron. Therefore, each velocity determines the deflection distance. Since the position of the detector (rectangular channeltron array with $5-10 \mathrm{~mm}$ width) is fixed, as shown in Fig. 1, the deflection of deuterium with low velocity becomes larger than that of proton. The deflec-

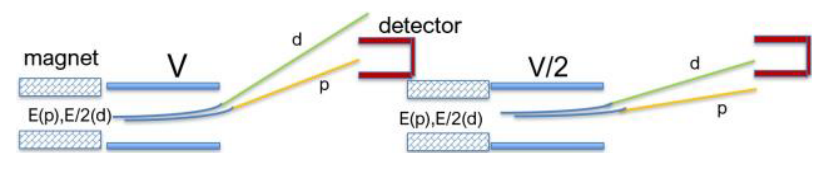

Fig. 1 Deflection Voltage.

Particle energies (proton, deuteron, etc.) are separated by the permanent magnet. A deuteron with $E / 2$ enters the same entrance of the deflection plates, where a proton with $E$ enters. If the plate voltage of $V$ reduces to be $V / 2$, the deuteron with $E / 2$ can be observed. In experiments, the plate voltage has been changed shot by shot in similar discharges.

tion voltage should be halved for deuterium measurement. Therefore, the proton orbit is between the equatorial plane and the detector due to half electric field.

Neutral particles (deuterium) pass through a pinhole diameter of $1 \mathrm{~mm} \varphi$ located at 2,397 $\mathrm{mm}$ from an entrance port $(30 \mathrm{~mm} \varphi)$ and are ionized by the carbon film. Since the deuterons travel about $350 \mathrm{~mm}$ from the pinhole to the detector, they spread to be $5 \mathrm{~mm} \varphi$ on the detector. Therefore, some protons may be mixed in the detector.

\section{Mass Rejection Factor}

The mass rejection factor means how many deuterons are mixed with one contamination proton in measuring deuterium. Larger values indicate better discrimination between proton and deuteron. A typical neutral particle analyzer has the mass rejection factor of several tens to 1,000 . Since CNPA is designed for hydrogen measurement, deuterons reach beyond the detector array. The position is far from the equatorial plane. Therefore, only protons can be detected. However, as shown in Fig. 1, protons arrive between the equatorial plane and the detector due to half electric field at adjusting deuteron measurement. This means the mass rejection factor will be reduced. However, a qualitative tendency in the deuterium/hydrogen ratio can be obtained.

Figure 2 shows a flux change when a deflection electric field is swept in a hydrogen/deuterium mixed plasma. The tail is 2 at $0.8 \mathrm{kV}$ when the peak value of $\mathrm{D}$ is 12 at $2.75 \mathrm{kV}$. This means that $\mathrm{D}$ contribution may be $1-2$ at $5.5 \mathrm{kV}$ assuming symmetry of the profile. Then $10-20 \%$ can be estimated from dividing $1-2$ by 12 . Therefore, protons of $10-20 \%$ are mixed when the deflection voltage is set for deuterium.

The mass rejection factor can be obtained by comparing two similar pure hydrogen plasma discharges. We can easily obtain the count ratio of D-channel and P-channel. As shown in Fig. 3, the mass rejection factor is high in the low energy region. The re-ionized particle in the thin foil is bent by the magnet. The ionized particle beam has the divergence angle. The flight distance in high energy particle is longer as shown in Fig. 3. The beam spread is larger in high energy region. Therefore, some of protons (deuteron) 


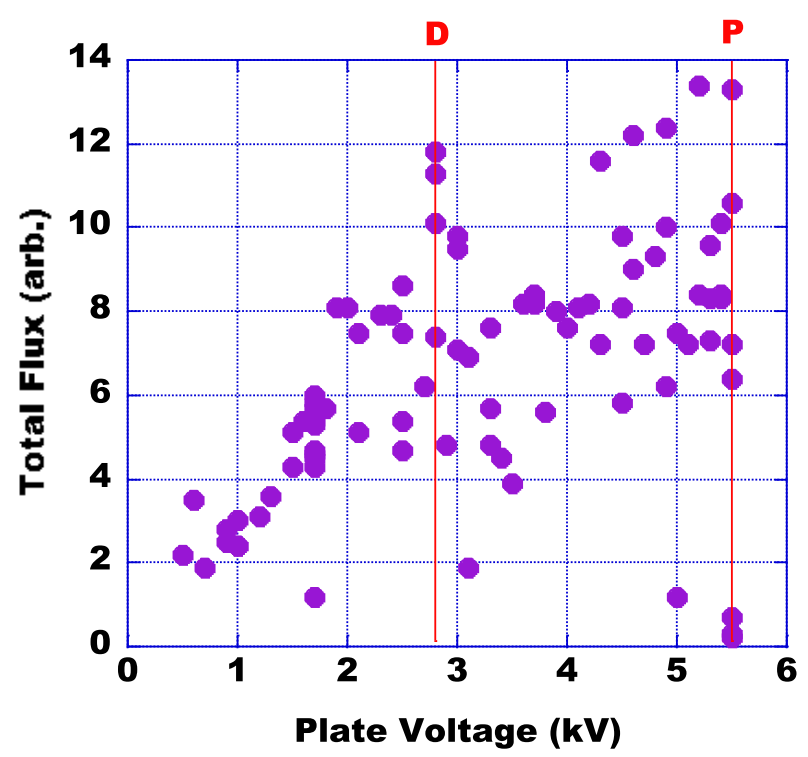

Fig. 2 Plate Voltage Scan.

Deflection plate voltage has been scanned during similar 100 discharges. When the voltage is set at " $\mathrm{D}$ " channel, some protons have been mixed.

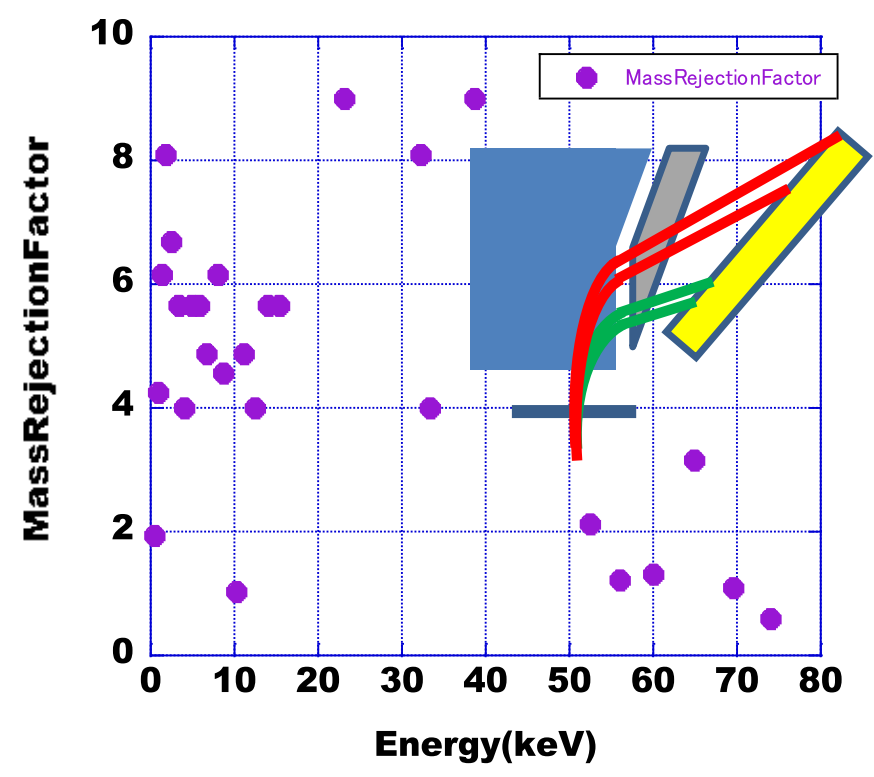

Fig. 3 Mass Rejection Factor.

The mass rejection factor is the number of deuterons per one noise proton. Mass rejection factor can be obtained from the ratio of $\mathrm{D} / \mathrm{H}$ at pure proton discharge.

enter the detector as contamination.

\section{Experimental Results}

Figure 4 (a) shows a time history of the $\mathrm{D} /(\mathrm{D}+\mathrm{H})$ ratio measured by CNPA in a typical hydrogen/deuterium mixed plasma discharge. Deuterium gas puff is used. The plasma is created with two perpendicular deuterium beams (DNBI), and maintained by three tangential hydrogen NBI

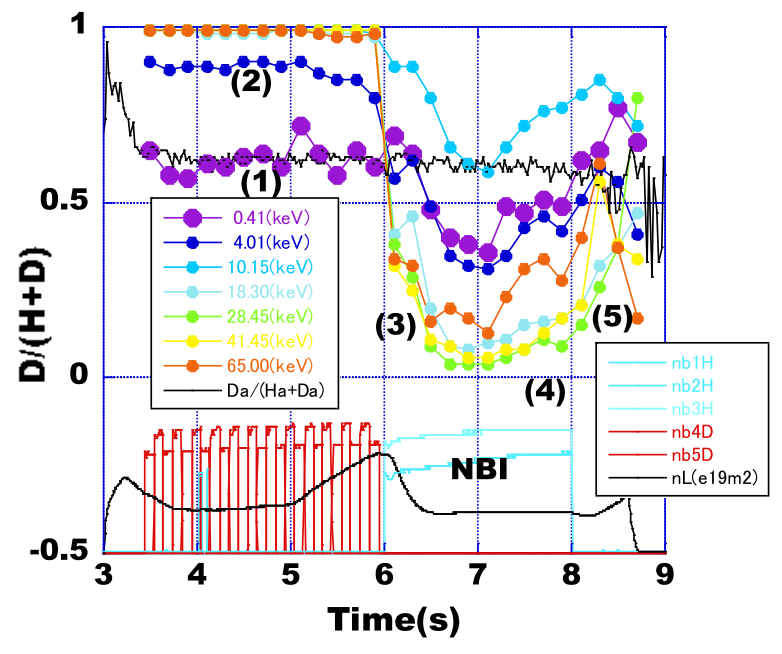

Fig. 4 (a) Time History of $D /(D+H)$.

Energy/time resolved $\mathrm{D} /(\mathrm{D}+\mathrm{H})$ has been observed during the D-NB and H-NB injections.

(1) Low energy components in D-NBI phase.

(2) High energy components in D-NBI phase.

(3) D-NBI off, (4) H-NBI on, (5) D and H-NBI off.

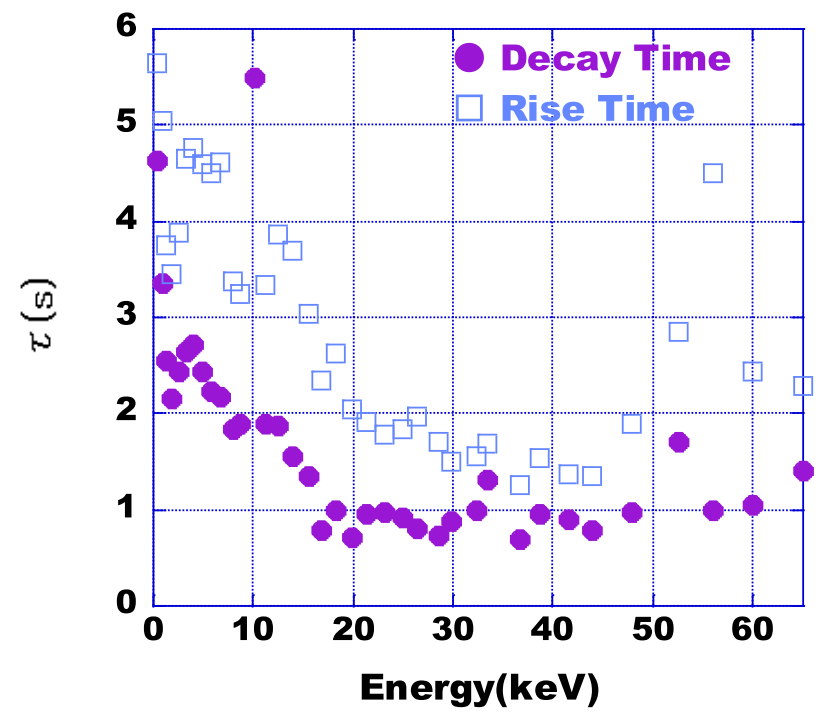

Fig. 4 (b) Decay and Rise Times of $D /(D+H)$. Decay and rise times of $D /(D+H)$ are obtained in $D$ NBI and H-NBI sustained plasma.

(H-NBI). The ratio is compared with $\mathrm{D}_{\alpha} /\left(\mathrm{D}_{\alpha}+\mathrm{H}_{\alpha}\right)$ measured by spectroscopy.

During D-NBI, $D /(D+H)$ corresponds to $D_{\alpha} /\left(D_{\alpha}+H_{\alpha}\right)$ at low energy region. This means that the low energy ions are localized in the peripheral region, which the spectroscopic measurement observes ((1) in Fig. 4 (a)). However, $\mathrm{D} /(\mathrm{D}+\mathrm{H})$ is quickly decreased due to the dilution by hydrogen although $\mathrm{D}_{\alpha} /\left(\mathrm{D}_{\alpha}+\mathrm{H}_{\alpha}\right)$ does not change during H-NBI phase ((3)). This suggests that the ratio measured by the spectroscopic method is strongly affected by hydrogen and deuterium adsorbed in the vacuum vessel wall. Decrease of $\mathrm{D} /(\mathrm{D}+\mathrm{H})$ during $\mathrm{H}-\mathrm{NBI}$ phase is faster than increase 
of $\mathrm{D} /(\mathrm{D}+\mathrm{H})$ during $\mathrm{D}-\mathrm{NBI}$ because light proton diffusion time is larger than heavy deuteron ((4)) [12].

In the high energy region, the time variation of the $\mathrm{D} /(\mathrm{D}+\mathrm{H})$ ratio strongly depends on NBI particle species. $\mathrm{D} /(\mathrm{D}+\mathrm{H})$ in the high energy region remains high by $\mathrm{D}$ dilution and lack of H-NBI during D-NBI phase. D/(D+H) decreases rapidly after D-NBI off and H-NBI on. However, $\mathrm{D} /(\mathrm{D}+\mathrm{H})$ gradually increases in the $\mathrm{H}-\mathrm{NBI}$ phase due to the collision of high energy protons with background D plasma (H-D collision). After H-NBI off, D/(D+H) increases again because there is no dilution of $\mathrm{H}$.

Figure $4(\mathrm{~b})$ shows the decay time and the rise $(\tau)$ during H-NBI phase assuming the exponential fitting in Fig. 4 (a). Both the decay time and the rise time are large at low energies. The large $\tau$ (= slow) at low energy region is due to $\mathrm{D}$ and $\mathrm{H}$ dilution from the wall. This indicates that the low-energy ions are localized in the peripheral region because the influence of absorbed neutrals from the wall is larger than the dilution by the particles supplied from the NBI. At $20-50 \mathrm{keV}$, the effect of the vacuum wall is small and the effect of dilution of the particles from NBI is large, thus $\tau$ is small and immediately decreases or increases.

$\mathrm{D} /(\mathrm{D}+\mathrm{H})$ increases during $\mathrm{H}-\mathrm{NBI}$ phase by the collision between $\mathrm{H}$ and $\mathrm{D}$ plasma, but the cross-section of $\mathrm{H}$ $\mathrm{D}$ collision is small in the high energy region. Therefore, During H-NBI phase, $\tau$ increases (= slow) at $>50 \mathrm{keV}$.

The rise time is slower than the decay time. This means that increasing by $\mathrm{H}-\mathrm{D}$ collisions during the $\mathrm{H}-\mathrm{NBI}$ phase is slower than dilution by H-NBI due to a complicated collision process.

\section{Time History of $\mathrm{D} /(\mathrm{D}+\mathrm{H})$}

The deuterium and the hydrogen neutral particles have been measured over several thousand shots by changing the plate voltage shot by shot. $\mathrm{D} / \mathrm{H}$ ratios could be obtained by comparing two similar discharges. Figure 5 shows the $\mathrm{D} /(\mathrm{D}+\mathrm{H})$ history over 2400 shots obtained from the energy integrated total flux. The $\mathrm{D}_{\alpha} /\left(\mathrm{D}_{\alpha}+\mathrm{H}_{\alpha}\right)$ obtained by a visible spectroscopy are also plotted in Fig. 5. In this estimation, low energy components are dominant because lower component is large in the CNPA. In the spectroscopic measurement, the contribution of low energy components in the peripheral region is also dominant. Therefore, both results are agreed in a wide range. Approximately 300 discharges are necessary to replace the deuterium plasma to the hydrogen plasma completely due to the large hydrogen/deuterium absorption in the wall.

\section{Summary}

The $\mathrm{D} /(\mathrm{D}+\mathrm{H})$ ratio in the plasma has a large effect on the behavior of the plasma. The measurement of this ratio using the charge exchange particle measurement is a

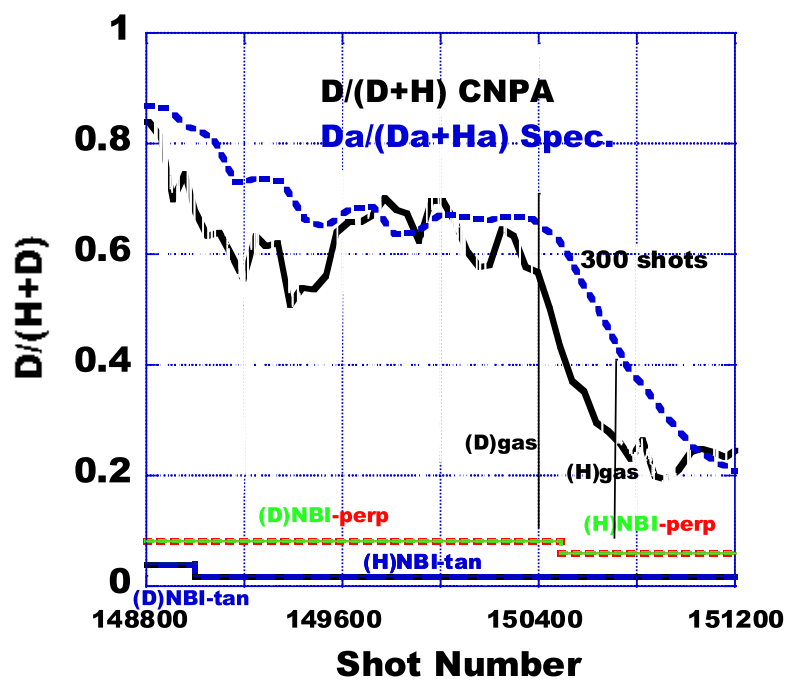

Fig. 5 D/(D+H) History During 2400 Shots.

Hydrogen glow discharge starts at the replace timing of fuel gas.

unique method especially at high energy region. The deflection voltage is changed for each shot to measure deuterium because CNPA is designed to measure only the energy distribution of hydrogen. The deuterium/hydrogen ratio is strongly affected by the inflow of hydrogen from the vacuum wall at low energy region, and is affected by dilution from NBI at high energy region. When the background gas is deuterium, high energy deuterium is observed by collision between $\mathrm{H}$ and $\mathrm{D}$. The behavior of the ratio in high and low energy regions can be explained by the classical slowing down process. Approximately 300 discharges are necessary to replace the deuterium plasma to the hydrogen plasma completely due to the large hydrogen/deuterium absorption in the wall.

\section{Acknowledgments}

Authors thank NIFS for financial support from code: NIFS ULRR006. We appreciate Dr. Motoshi Goto for providing spectroscopic data.

[1] O. Motojima et al., Fusion Eng. Des. 20, 3 (1993).

[2] S. Morita et al., Plasma Fusion Res. 3, S1037 (2008).

[3] K. Yamasaki et al., Plasma Fusion Res. 13, 1202103 (2018).

[4] P. Goncharov et al., Rev. Sci. Instrum. 79, 10F312 (2008).

[5] T. Ozaki et al., J. Plasma Fusion Res. 8, 1089 (2009).

[6] S. Sudo et al., Rev. Sci. Instrum. 83, 023503 (2012).

[7] T. Ozaki et al., Plasma Fusion Res. 7, 2402138 (2012).

[8] S. Medley et al., Rev. Sci. Instrum. 69, 2651 (1998).

[9] M. Isobe et al., Rev. Sci. Instrum. 85, 11E114 (2014).

[10] T. Nishitani et al., Fusion Eng. Des. 136, 210 (2018).

[11] T. Ozaki et al., Plasma Fusion Res. 14, 3402142 (2019).

[12] H. Takahashi et al., Nucl. Fusion 58, 106028 (2018). 